ИЗВЕСТНЯ АКАДЕМИИ НАУК ЭСТОНСКОИ ССР. ФИЗИКА * МАТЕМАТИКА

PROCEEDINGS OF THE ACADEMY OF SCIENCES OF THE ESTONIAN SSR. PHYSICS * MATHEMATICS

$1986, \quad 35,1$

\title{
ОБ ОДНОЗНАЧНОСТИ СООТВЕТСТВИЯ МЕЖДУ УРАВНЕНИЕМ И ЕГО ГРУППОЙ ИНВАРИАНТНОСТИ В СЛУЧАЕ ВЫСШИХ РАЗМЕРНОСТЕЙ
}

\author{
(Представил Х. Керес)
}

\begin{abstract}
Изучается однозначность восстановлення вида уравнения по его наперед заданной группе инвариантности в случае, когда число переменных многообразия (зависимых и независимых) больше трех. Исходная группа инварнантности выбирается с учетом принципа равноправия полевых и пространственных переменных. Показано существование группы симметрии, приводящей однозначно к уравнению, ее допускающему. В случае единственной функцин многих переменных такое уравнение описывает поверхности с нулевой полной кривизной.
\end{abstract}

Проблема нахождения группы точечных преобразований для данного дифференциального уравнения в частных производных (д.у.ч.п.) подробно освещена в литературе (см., напр., [1 $\left.\left.{ }^{1}\right]\right)$. Изучены также группы касательных преобразований и группы Ли-Бэклунда $\left[{ }^{2}\right]$ некоторых интересных уравнений. Предложен и алгоритм решения обратной задачи - восстановления вида уравнения по его группе инвариантности $\left[{ }^{3}\right]$.

В предыдущей работе $\left[{ }^{4}\right]$ была поставлена и решена проблема однозначности такого восстановления в случае одной функции двух переменных и показано, что уравнение Монжа-Ампера для поверхности $u=$ $=u(x, y)$ с нулевой гауссовой кривизной

$$
u_{x x} u_{y y}-u_{x y}{ }^{2}=0
$$

находится во взаимно-однозначном соответствии со своей 15-параметрической группой точечных преобразований.

В данной работе будет продолжено изучение однозначности соответствия между уравнением и его группой в случае, когда число всех переменных многообразия (зависимых и независимых) больше трех. Как и в $\left[{ }^{4}\right]$, при выборе группы будем руководствоваться соображениями полного равноправия полевых и пространственных переменных (принцип годограф-инвариантности) $\left[{ }^{5,6}\right]$, что выражается в полной симметричности исходной группы относительно всех своих переменных.

Пусть $n_{f}$ и $n_{x}$ - число функций и аргументов соответственно.

1. Пусть $n_{f}=1, n_{x}=n$.

Рассмотрим группу $G$ на $(n+1)$-мерном многообразии: $x_{1}, \ldots, x_{n}, \varphi$.

Базис соответствующей алгебры Ли группы $G$ зададим в виде всевозможных генераторов

$$
\frac{\partial}{\partial a}, \quad a \frac{\partial}{\partial a}, \quad a \frac{\partial}{\partial b}
$$

где $a, b$ - переменные многообразия (в соответствии с требованием полной симметрии относительно всех переменных деления на зависимые и независимые переменные не производится). 
Будем искать теперь $\left[{ }^{2}\right]$ класс уравнений II порядка, допускающих данную группу $G$. Инфинитезимальный оператор группы $G$ :

где согласно (2)

$$
X=\xi^{i} \frac{\partial}{\partial x_{i}}+\eta \frac{\partial}{\partial \varphi},
$$

$$
\begin{aligned}
& \xi^{k}=A_{0}^{k}+A_{i}^{k} x_{i}+A_{n+1}^{k} \varphi, \\
& \eta=B_{0}+B_{i} x_{i}+B_{n+1} \varphi,
\end{aligned}
$$

$A_{\mu}^{k}, B_{\mu}-$ групповые параметры,

$$
\mu=0,1, \ldots, n+1, \quad k=1,2 ; \ldots, n .
$$

Инфинитезимальный оператор дважды продолженной группы определяется из соотношений $\left[{ }^{1}\right]$

$$
\begin{aligned}
\widetilde{\widetilde{X}} & =X+\zeta_{i} \frac{\partial}{\partial p_{i}}+\sigma_{i j} \frac{\partial}{\partial r_{i j}}, \\
\zeta_{i} & =D_{i}(\eta)-p_{j} D_{i}\left(\xi^{j}\right), \\
\sigma_{i j} & =\left(D_{i}+r_{i k} \frac{\partial}{\partial p_{k}}\right) \zeta_{j}-r_{i k} D_{j}\left(\xi^{k}\right), \\
D_{i} & =\frac{\partial}{\partial x_{i}}+p_{i} \frac{\partial}{\partial \varphi} \\
p_{i} & \equiv \frac{\partial \varphi}{\partial x_{i}}, \quad r_{i j} \equiv \frac{\partial^{2} \varphi}{\partial x_{i} \partial x_{j}} \equiv \varphi_{i j} .
\end{aligned}
$$

Если теперь в выражении для $\widetilde{\widetilde{X}}$ собрать коэффициенты перед различными параметрами группы $A_{\mu}{ }^{k}, B_{\mu}$, то получим генераторы дважды продолженной группы $\widetilde{\widetilde{G}}: \widetilde{\mathfrak{F}}_{\alpha}$, сответствующие непродолженным генераторам (2).

Уравнения, допускающие группу $G$, запишем в виде

$$
\begin{gathered}
h\left(\left\{x_{i}\right\}, \varphi,\left\{p_{i}\right\},\left\{r_{i j}\right\}\right)=0 \\
i, j=1 ; 2, \ldots, n .
\end{gathered}
$$

Тогда для нахождения явного вида $h$ потребуем выполнения следующих условий

$$
\left.\tilde{\mathfrak{x}}_{\alpha} h\right|_{h=0}=0, \quad \forall \alpha .
$$

(Вообще говоря, достаточно потребовать выполнения (6) лишь для тех $\widetilde{\dddot{x}}_{\alpha}$, для которых соответствующие $\widetilde{\tilde{ঋ}}_{\alpha}$ генерируют всю алгебру $\left.(2)\right)$.

Опуская длинные и трудоемкие, но прямолинейные вычисления, приведем систему уравнений для искомой функции $h$ (уравнения $h=0$ ):

$$
\begin{gathered}
h=h\left(\left\{r_{i j}\right\}\right), \\
i, j=\mathrm{fix}, \quad \sum_{l \leqslant j} r_{i l} h_{l j}+\left.\sum_{l \geqslant j} r_{i l} h_{l j}\right|_{h=0}=0, \\
\left.\sum_{i \leqslant j} r_{i j} h_{i j}\right|_{h=0}=0, \\
i, j, l=1, \ldots, n ; \quad h_{i j} \equiv \frac{\partial h}{\partial r_{i j}} .
\end{gathered}
$$


Если выписать первые $n$ уравнений этой системы

$$
\begin{aligned}
& 2 \varphi_{11} h_{11}+\varphi_{12} h_{12}+\varphi_{13} h_{13}+\ldots+\left.\varphi_{1 n} h_{1 n}\right|_{h=0}=0, \\
& 2 \varphi_{21} h_{11}+\varphi_{22} h_{12}+\varphi_{23} h_{13}+\ldots+\left.\varphi_{2 n} h_{1 n}\right|_{h=0}=0, \\
& \left.2 \cdot \cdot \cdot \cdot \cdot \cdot \cdot \cdot \cdot \cdot \cdot \cdot \cdot \cdot \cdot \cdot \cdot \cdot \cdot \cdot \cdot \cdot \cdot \cdot \cdot \cdot \cdot \cdot \cdot \varphi_{n n} h_{1 n}\right|_{h=0}=0,
\end{aligned}
$$

то можно легко заключить, что единственно возможный вид $h=0$ :

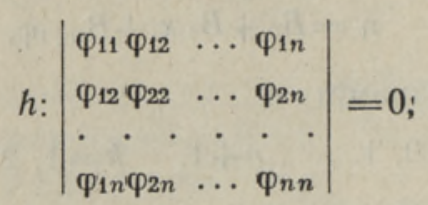

так как (9) - условие непротиворечивости системы (8).

Нетрудно проверить, что (9) действительно задает искомую $h$ и удовлетворяет всем уравнениям системы (7). Рассмотрим, например, уравнение

$$
2 \varphi_{11} h_{11}+\varphi_{12} h_{12}+\varphi_{13} h_{13}+\ldots+\varphi_{1 n} h_{1 n}=0 .
$$

Левая часть этого уравнения с учетом (9) имеет вид

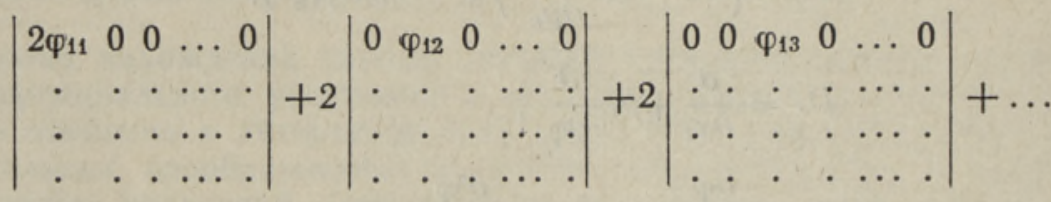

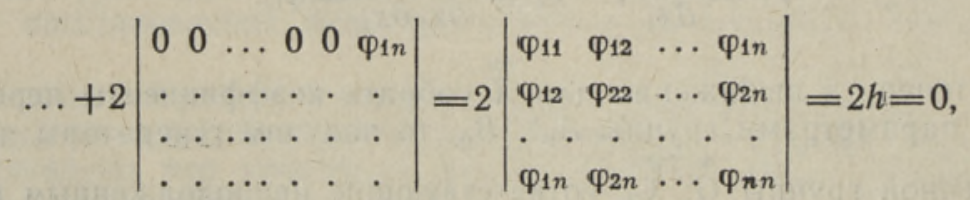

где точками обозначены соответствующие элементы детерминанта (9). Аналогичным образом показывается справедливость остальных уравнений системы (7).

Таким образом, группа $G$, заданная генераторами (2), однозначно приводит к уравнению (9).

За меч ан ие 1. Рассматриваются лишь д.у.ч.п. II порядка, а тривиальные уравнения вида $\varphi_{i j}=0$ исключаются из рассмотрения.

3 а м е ч н и е 2. Уравнение (9) исследовалось А. В. Погореловым [ $\left.{ }^{7}\right]$ в связи с решением многомерной проблемы Минковского. Группа симметрии уравнения (9) и некоторые точные решения были получены B $\left[{ }^{8}\right]$.

3 а меч ан и е 3 . Уравнение (9) в работе [ $\left.{ }^{4}\right]$ было пропущено.

3 а м ч а н и 4 . На самом деле уравнение (9) обладает более богатой, чем задано выражением (2), группой точечных преобразований: к (2) добавляются генераторы вида

$$
\begin{gathered}
\mathfrak{X}_{\mu}=a D \\
D=\sum_{i=1}^{n} x_{i} \frac{\partial}{\partial x_{i}}+\varphi \frac{\partial}{\partial \varphi} .
\end{gathered}
$$

Можно легко показать, что уравнение (9) описывает поверхность $\varphi=\varphi\left(x_{1}, \ldots, x_{n}\right)$ с полной кривизной $K=0$. 
Действительно, для главных кривизн $k_{i}(i=1,2, \ldots, n)$ имеем следующее уравнение $\left[{ }^{9}\right]$

$$
\left|b_{i j}-k g_{i j}\right|=0
$$

или

$$
(-k)^{n}\left|g_{i j}\right|+(-k)^{n-1} R_{1}+\ldots+(-k) R_{n-1}+\left|b_{i j}\right|=0,
$$

где $R_{b}(b=1, \ldots, n-1)$ - некоторые функции $b_{i j}, g_{i j}$,

$$
\begin{aligned}
& g_{i j}=\vec{r}_{i} \cdot \vec{r}_{j}, \\
& b_{i j}=\vec{r}_{i j} \cdot \vec{m}
\end{aligned}
$$

коэффициенты I и II основных квадратичных форм соответственно,

$$
\begin{aligned}
& \vec{r}=\left(x_{1}, x_{2}, \ldots ; x_{n}, \varphi(x)\right), \\
& \overrightarrow{r_{i}}=\frac{\partial \vec{r}}{\partial x_{i}}, \quad \vec{r}_{i j}=\frac{\partial^{2} \vec{r}}{\partial x_{i} \partial x_{j}}, \\
& \vec{m} \cdot \vec{r}_{i}=0, \quad \forall i ; \quad|\vec{m}|=1 .
\end{aligned}
$$

Запишем выражение для $\vec{m}$ с помощью косого произведения

$$
\vec{m} \sim\left[\vec{r}_{1}, \vec{r}_{2}, \ldots ; \vec{r}_{n}\right]
$$

Тогда

$$
b_{i j}=\frac{\vec{r}_{i j}\left[\vec{r}_{1}, \vec{r}_{2}, \ldots, \vec{r}_{n}\right]}{\left|\left[\vec{r}_{1}, \ldots, \vec{r}_{n}\right]\right|}
$$

и в результате получим

$$
b_{i j} \sim \varphi_{i j}
$$

Отсюда для полной кривизны с учетом $\left(11^{\prime}\right)$

$$
K=\prod_{i=1}^{n} k_{i}=(-1)^{n} \frac{\left|b_{i j}\right|}{\left|g_{i j}\right|} \sim\left|\varphi_{i j}\right|,
$$

что означает, что уравнение (9) $\left(\left|\varphi_{i j}\right|=0\right)$ эквивалентно условию $K=0$. C этой точки зрения переопределенная система уравнений

$$
h^{\prime}: \varphi_{i k} \varphi_{l m}-\varphi_{i l} \varphi_{k m}=0,
$$

также являющаяся решением системы (7), представляет из себя частный случай уравнения (9) и соответствует $K=0$ и

$$
k_{1}=k_{2}=\ldots=k_{n-1}=0 ; \quad\left(k_{n}=\frac{b_{i j} g^{i j}}{\operatorname{det} g}\right) .
$$

2. $n_{f}=2, n_{x}=2$.

Как и в случае 1 зададим исходную группу инвариантности на 4-мерном многообразии $V^{4}: x_{1}, x_{2}, \varphi^{1}, \varphi^{2}$ всевозможными генераторами вида (2), но с $a, b \subset V^{4}$. 
Описанный выше алгоритм восстановления вида уравнения по его группе приводит к следующим уравнениям на искомую функцию

$$
\begin{gathered}
i, j=\mathrm{fix},\left.\quad \sum_{\alpha}\left(\sum_{l \leqslant j} r_{i l}^{\alpha} h_{l j}^{\alpha}+\sum_{l \geqslant j} r_{i l}^{\alpha} h_{l j}^{\alpha}\right)\right|_{h=0}=0 \\
\sum_{i \leqslant j}^{\left.r_{i j}^{\alpha} h_{i j}^{\beta}\right|_{h=0}=0} \\
h=h\left(\left\{r_{i j}^{\alpha}\right\}\right), \quad r_{i j}^{\alpha} \equiv \frac{\partial^{2} \varphi^{\alpha}}{\partial x_{i} \partial x_{j}} \\
i, j, l, \alpha ; \beta=1,2 .
\end{gathered}
$$

Можно показать, что система (15) имеет единственное нетривиальное решение

$$
\begin{gathered}
h:\left(\varphi_{11}^{1} \varphi_{22}^{2}-\varphi_{22}^{1} \varphi_{11}^{2}\right)^{2}+4\left(\varphi_{11}^{1} \varphi_{12}^{2}-\varphi_{12}^{1} \varphi_{11}^{2}\right)\left(\varphi_{22}^{1} \varphi_{12}^{2}-\varphi_{12}^{1} \varphi_{22}^{2}\right)=0 \\
\varphi_{i j}^{\alpha} \equiv \frac{\partial^{2} \varphi^{\alpha}}{\partial x_{i} \partial x_{j}} .
\end{gathered}
$$

3 а меч а н и 5 . Мы исключили из рассмотрения частное (тривиальное) решение (15):

$$
h^{\prime}: \frac{\varphi_{11}^{1}}{\varphi_{11}^{2}}=\frac{\varphi_{12}^{1}}{\varphi_{12}^{2}}=\frac{\varphi_{22}^{1}}{\varphi_{22}^{2}}=l .
$$

Имеем

$$
\left(\frac{\varphi_{11}^{1}}{\varphi_{11}^{2}}\right)_{, 2}=\left(\frac{\varphi_{12}^{1}}{\varphi_{12}^{2}}\right)_{, 1} \cdot \frac{\varphi_{12}^{2}}{\varphi_{11}^{2}}
$$

и

$$
\left(\frac{\varphi_{22}^{1}}{\varphi_{22}^{2}}\right)_{, 1}=\left(\frac{\varphi_{12}^{1}}{\varphi_{12}^{2}}\right)_{, 2} \cdot \frac{\varphi_{12}^{2}}{\varphi_{22}^{2}}
$$

откуда следует

$$
l=\text { const. }
$$

3 а меч ан и е 6 . Как и в случае 1 группа симметрии полученного уравнения (16) шире, чем задано выражением (6), а именно, к генераторам (2) следует добавить генераторы

$$
\begin{gathered}
\mathfrak{x}_{\mu}=a D \\
D=\sum_{i=1}^{2}\left(x_{i} \frac{\partial}{\partial x_{i}}+\varphi^{i} \frac{\partial}{\partial \varphi^{i}}\right) .
\end{gathered}
$$

Таким образом, мы показали, что однозначность соответствия между уравнением и его группой инвариантности для систем с полной пространственно-полевой симметрией имеет место и в случае высших размерностей, что, как показано в [ $\left.{ }^{4}\right]$, значительно отличает данные системы от нелинейных уравнений, обладающих симметрией иного вида.

Автор весьма признателен $M$. Кыйву за многочисленные обсуждения данной работы, а также Ю. Лумисте за объяснение геометрических аспектов рассматриваемых уравнений. 
1. Овсянников Л. В. Лекции по теории групповых свойств дифференциальных ұравнений. Новосибирск, «Наука», 1966.

2. Ибрагимов H. X. Группы преобразований в математической физике. М., «Наука», 1983.

3. Bluman, G. W., Cole, I. D. Similarity methods for differential equations. - Appl. Math. Sci., 13 (1974).

4. Розенгауз В. Препринт F-18, Тарту, 1982.

5. Айнсаар А., Кийранен $K$., Кыйв М. Изв. АН ЭССР. Физ. Матем., 27, № 4, 453455 (1978); Кьий М., Розенгауз В. Изв. АН ЭССР. Физ. Матем., 28, № 3, 187-193 (1979).

6. Шварц А. С. Препринт ITEP-2 Ин-та теор. эксп. физ., М., 1980; Гайдук А. В., Романов В. Н., Шварц А. С. Препринт ITЕР-36 Ин-та теор. эксп. физ, М., 1980,

7. Погорелов А. В.Многомерная проблема Минковского. М., «Наука», 1975.

8. Фуици В. И., Серов Н. И. Докл. АН СССР, 273, 543-546 (1983).

9. Эйзенхарт Л. П. Риманова геометрия. М., ИЛ, 1948.
Институт физики
Академии наук Эстонской ССР
Поступила в редакцию
6/XII 1984

\section{ROSENHAUS}

\section{VORRANDI JA TEMA INVARIANTSUSE ROHMA VASTAVUSE OHESUSEST KORGEMATE DIMENSIOONIDE JUHUL}

Artikkel käsitleb võrrandi kuju taastamise ühesust etteantud invariantsuse rühma abil, juhul kui muutkonna muutujate (sõltuvate ja sõltumatute) arv on kolmest suurem. On lähtutud invariantsuse rühmast, mis valitakse ruumi ja välja sümmeetria printsiibi alusel. On näidatud, et eksisteerib sümmeetria rühm, mis üheselt määrab vastava vôrrandi. Uheainsa mitme muutuja funktsiooni puhul selline võrrand kirjeldab null täiskõverusega pinda.

\section{ROSENHAUS}

\section{ON THE UNIQUENESS OF THE CORRESPONDENCE BETWEEN THE EQUATION AND ITS INVARIANCE GROUP IN HIGHER DIMENSIONS}

The paper deals with the uniqueness of the construction of an equation by its given invariance group when the number of manifold variables (dependent and independent ones) is greater than three. The field-space equality principle is taken into account in choosing the initial group of invariance. A symmetry group leading uniquely to the equation admitting it, is shown to exist. In the case of only one function of many variables such an equation describes the surfaces with zero total curvature. 